\title{
First Design of High Birefringence and Polarising Hollow Core Anti-resonant Fibre
}

\author{
S.A. Mousavi(1), D.J. Richardson(1), S.R. Sandoghchi(1), F. Poletti ${ }^{(1)}$ \\ (1) Optoelectronics Research Centre, University of Southampton, Southampton SO17 1BJ, United \\ Kingdom, sam1e12@soton.ac.uk
}

\begin{abstract}
We thoroughly study possible ways to introduce high birefringence and differential loss in hollow core anti-resonant fibres. We present the design of realistically achievable fibres with large birefringence and effectively polarising behaviour over the entire C-band.
\end{abstract}

\section{Introduction}

High birefringence (Hi-Bi) fibres are widely used in optical communication systems, sensing and interferometric devices, where preserving the source polarisation is essential. In solid core polarization-maintaining (PM) fibres, the $\mathrm{Hi}-\mathrm{Bi}$ effect is achieved by applying ether stress or anisotropy (elliptic core) to the fibre ${ }^{1}$ but the fibre nonlinearity and/or its sensitivity to external perturbations like temperature can limit the performance of the final devices they are used in.

Due to their unique low nonlinearity and high insensitivity to thermal environmental variations, hollow-core $(\mathrm{HC})$ fibres could represent an attractive alternative for use within high precision sensors, e.g. in gas spectroscopy or in fibre optic gyroscopes (FOG) ${ }^{2}$. However, high performance, low-loss $\mathrm{HC}$ with polarisation maintaining properties are not readily available. Some PM HC fibres based on photonic band gap (PBG) guidance have been presented recently ${ }^{3}$. Due to their different guiding mechanism, the PM property is typically achieved by exploiting the anti-crossing of coreclad modes instead of the conventional methods of solid core fibres. However, these PM HC fibres have a typically fairly high propagation loss and their operation is sensitive to external perturbations.

In this paper, we propose both a PM and a polarizing $(\mathrm{PM} / \mathrm{P}) \mathrm{HC}$ fibre based, for the first time, on anti-resonance guidance. The underlying structure, based on a recently proposed ultra-low loss nested anti-resonance nodeless (NANF) design ${ }^{4}$, allows greater control of the structural parameters than PBG fibres and ultimately offers improved PM properties. We found an optimized fibre operating at a wavelength of $1.55 \mu \mathrm{m}$ with a phase birefringence (PB) as high as $1.4 \times 10^{-4}$, a loss for the lowest-loss polarization of $0.075 \mathrm{~dB} / \mathrm{m}$ and a 1000 times higher loss for the other polarization, which makes the $\mathrm{HC}$ fibre effectively polarising after only a very short propagation length.

\section{Asymmetric NANF (elliptical mode shape in NANF)}

The proposed ultra-low loss NANF in ${ }^{4}$ is a tubular structure consisting of thin nested tubes attached to the inner wall of a larger capillary in such a way that they do not touch each other. This design reduces the confinement loss (CL) due to the use of extra reflecting antiresonance layers from the additional inner tubes and to the elimination of glass nodes in the clad structure. Although the proposed NANF has shown very low loss profile, potentially comparable to solid telecoms fibres and has broadband transmission window ${ }^{4}$, its symmetrical structure supports degenerate fundamental modes and cannot maintain light a)

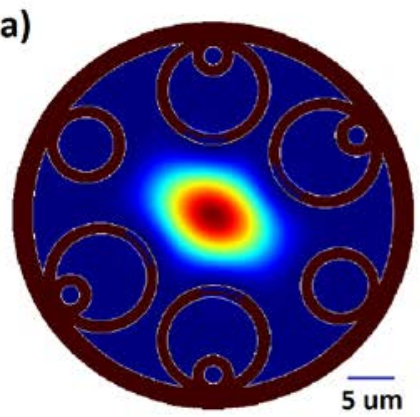

b)

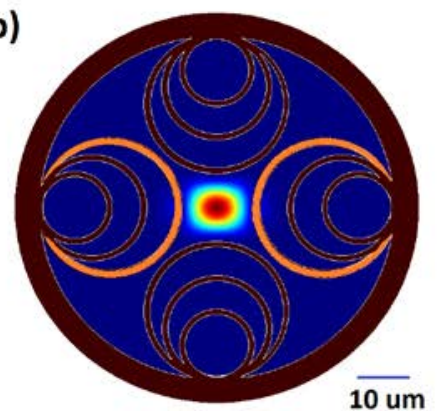

c)

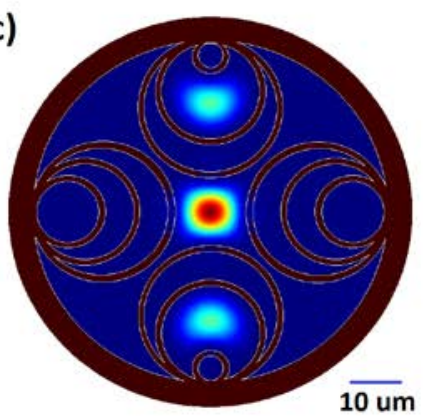

Fig. 1: a) An elliptical mode shape in NANF, b) PB in NAFN by mode anti-crossing due to thickness difference (orang ring is thicker tube), c) Hiqh loss in NANF due to core-to-clad coupling effect. 


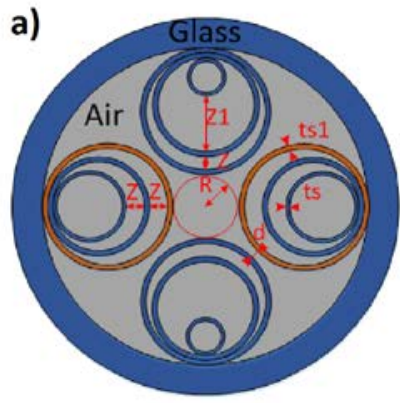

b)

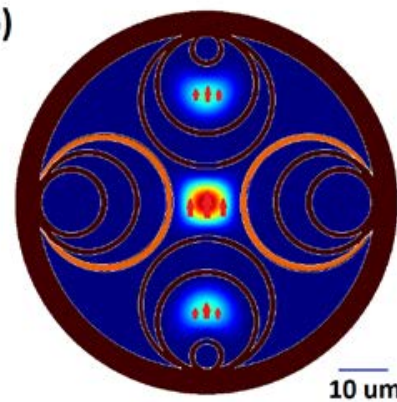

c)

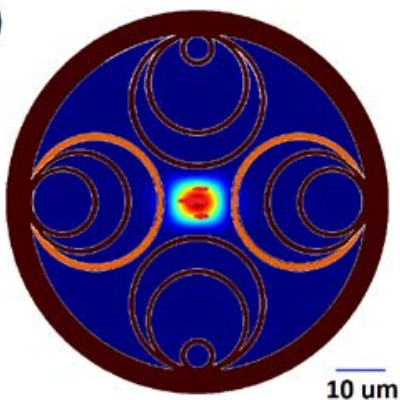

Fig. 2: Proposed HC PM/P NANF, a) Structural parameters, b) high-loss polarization mode, c) low-loss polarization mode (orang ring is thicker tube).

polarisation. In order to break the symmetry in this structure, as our first attempt, we tried following the strategy of elliptic core in solid core fibres, and modified the core shape of a six-tube NANF to an elliptic shape by reducing the size of the cladding tubes in one direction (Fig. 1.a). All simulations in this paper has been performed by a full vector finite-element based modal solver (COMSOL Multiphysics $®$ ) and using a perfectly matched layer (PML) with standard cylindrical definition ${ }^{5}$.

Although the structure shows a strongly elliptic mode profile, fibres with reasonably low loss could only achieve a PB for orthogonal polarizations of the fundamental mode (OPFM) of $\sim 3 \times 10^{-6}$ at $1.55 \mu \mathrm{m}$, which is approximately two orders of magnitude lower than commercially available solid core PM fibres $\left(\sim 10^{-4}\right)$. This low $\mathrm{PB}$ value is due to the strong dependence of the effective refractive index of $\mathrm{HC}$ anti-resonance waveguide on their core size. For instance, simulation shows that $P B$ in the range of $10^{-5}$ at wavelength of $1.55 \mu \mathrm{m}$ can be achieved in a simple anti-resonant fibre formed by a single elliptical silica tube nested in a thicker outer jacket, only if the core sizes are 3 and $2 \mu \mathrm{m}$ for semi-large and semi-small axis, respectively. However, with such a small core size the anti-resonance mechanism cannot prevent the field effectively from leaking out and the CL increases to more than $400 \mathrm{~dB} / \mathrm{m}$ in this case. Despite the $\mathrm{CL}$ reduction achievable in NANFs by adding extra inner tube, the necessity of extremely small elliptical core size to achieve acceptable PB $\left(10^{-4}\right)$, introduces an impractical high loss $(\sim 200 \mathrm{~dB} / \mathrm{m})$ in these fibres. Thus we conclude that elliptical core is not a practical solution for $\mathrm{HC}$ anti-resonance fibres.

\section{Mode anti-crossing PB effect in NANF}

The rapid and significant change in effective index of an air-guided mode at the edges of the anti-resonant frequency window has been used in $P B G$ fibres to introduce $P B$ in previous works $^{3}$. Here, we use a similar effect to introduce an effective index difference in the
OPFM of a four- tube NANF. Fig.1.b shows the proposed structure, consisting of four outer tubes with two inner tubes each, and its fundamental mode profile. In this structure, the thickness of all tubes is designed for operation at anti-resonance $\left(t_{s}=1.172 \mu \mathrm{m}\right)$, except for two of the outer tubes in one direction (shown in orange) that are designed to work at the edge of their resonance $\left(t_{s 1}=1.42 \mu \mathrm{m}\right)$ to introduce an effective index difference in that direction.

Here we chose a four rather than six-tube structure in order to try and achieve the smallest possible core with the largest possible separation to the outer jacket to minimise CL. By adding an extra nested tube the $\mathrm{CL}$ can be reduced significantly. Using a core inner diameter of $7 \mu \mathrm{m}$ and other structural parameters discussed in more detail later on for the fibre in Fig.3, our optimised fibre achieves a $P B=$ $1.54 \times 10^{-4}$, comparable to that of conventional solid PM fibres, and a loss of $\sim 0.01 \mathrm{~dB} / \mathrm{m}$ at $1.55 \mu \mathrm{m}$, which is remarkable for a $\mathrm{HC}$ fibre with such a small core.

We then went one step further, looking for designs that can also offer a high differential loss between polarisations. The idea is to optimize the radius of the clad tubes to achieve phase matching between core and clad guided modes and thus out couple core-fields into the cladding introducing large losses in the process. The idea was introduced to outstrip all high order modes in a symmetric NANF"; here we adapt it to operate on one of the fundamental modes only. Fig. 1.c shows a fibre where all tube thicknesses are the same. In this case both polarisations of the fundamental mode experience high loss $(\sim 50 \mathrm{~dB} / \mathrm{m})$ and no birefringence can be achieved.

The solution we propose is thus to combine the anti-crossing PB effect of the fibre in Fig. $1 \mathrm{~b}$ with the core-clad coupling loss effect of Fig. 1c. The result is a HC NANF with PM and polarizing (PM/P) ability. Fig. 2.a shows a schematic of the proposed $\mathrm{PM} / \mathrm{P}$ fibre based on a four-tube NANF structure including thicker outer cladding 

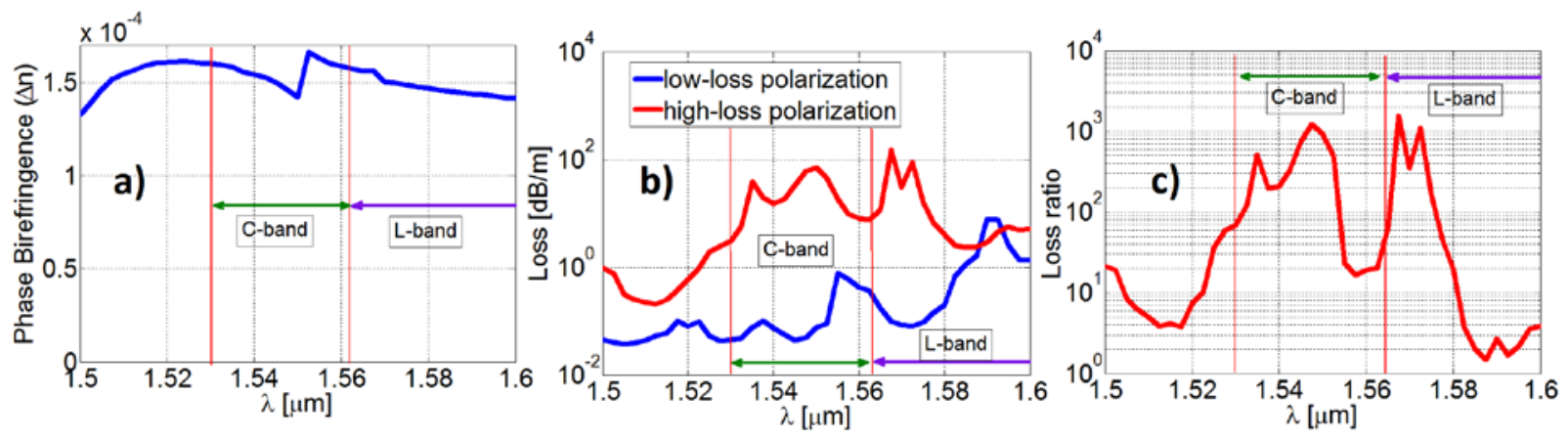

Fig. 3: Characteristics of proposed HC PM/P NANF optimized at $1.55 \mu \mathrm{m}$. a) PB, b) Loss, c) Loss ratio of the fibre for wavelengths between 1.5 to $1.6 \mu \mathrm{m}$. The fibre shows fairly flat PB and high loss ratio in C-band toward first half of the L-band.

tubes in one direction and clad-coupling mechanism in the other direction obtained by changing the inner tubes distance $\left(Z_{1}\right)$. These modification are responsible to introduce the PM and polarizing effect, respectively. Although the proposed structure can be designed for any desirable operating wavelength, we have optimized it here for $1.55 \mu \mathrm{m}$ operation. A core radius of $R=7 \mu m$ enables strong $\mathrm{PM}$ properties, low loss and a mode field diameter close to that of standard fibres. The other optimum parameter values to achieve maximum PB and differential loss between OPFM at 1.55 $\mu \mathrm{m}$ are: $Z=0.65 R, Z_{1}=1.74 R, d=1.5 \mu \mathrm{m}$, $t_{s}=1.172 \mu \mathrm{m}$ and $t_{s 2}=1.42 \mu \mathrm{m}$. Fig. 2.b and 2.c show the modal intensity profile and the electric field direction of the high-loss (y-polarised) and low-loss (x-polarised) OPFM at $1.55 \mu \mathrm{m}$, respectively.

In this fibre, the slightly different mode size for one polarization, arising from the different tube thickness, prevents coupling to cladding modes and maintains the loss as low as in a normal NANF. In the perpendicular polarization in contrast, the core-to-clad out-coupling process introduces a much larger loss, as shown in Fig. 3. The figure shows the PB, loss and loss ratio of OPFM for the optimised fibre for wavelengths between 1.5 and $1.6 \mu \mathrm{m}$. In this range the $P B$ value shows again values comparable to conventional PM fibres, with a small variation between $1.4 \times 10^{-4}$ to $1.7 \times$ $10^{-4}$. The sharp change around $1.55 \mu \mathrm{m}$ is due to a mode anti-crossing that we believe can be eliminated with further refinements. The lowest loss polarization has a loss as low as $\sim 0.08 \mathrm{~dB} / \mathrm{m}$. The $\sim 7$ times increase in loss as compared to the PM fibre in Fig. $1 \mathrm{~b}$ is counterbalanced by a much higher loss for the other polarization, $\sim 70 \mathrm{~dB} / \mathrm{m}$ at $1.55 \mu \mathrm{m}$. This would be sufficient to make this fibre truly single polarisation even in metre-scale applications, independently on the input polarisation state. As shown in Fig. 3, despite the fact they are based on resonant effects, the optical properties of these fibre have a fairly broad spectral range: the large birefringence, the low fundamental mode loss and the large loss differential extend across the entire $C$ band and to the first half of the $L$ band.

\section{Conclusions}

We have proposed what is, to the best of our knowledge, the first $\mathrm{HC}$ anti-resonant fibre design showing simultaneously polarizationmaintaining and polarizing ability. By carefully exploiting resonances from membranes of two different thicknesses and phase-matched modal outstripping effects, the optimized design for $1.55 \mu \mathrm{m}$ operation shows a phase birefringence as high as $1.4 \times 10^{-4}$. Besides, one polarisation of the fundamental mode has a loss of $0.076 \mathrm{~dB} / \mathrm{m}$, while the orthogonal polarisation has a $\sim 1000$ times higher loss, giving the fibre a polarising behaviour. The PM ability and the large differential loss of the fibre are broadband and extend well beyond the $\mathrm{C}$ band. Besides, Due to scalability of the design, it is possible to scale the proposed structures to other desired operating wavelength and achieve similar performances. A simpler version of the $\mathrm{HC}$ fibre with just PM ability but lower loss $(0.01 \mathrm{~dB} / \mathrm{m}$ at $1.55 \mu \mathrm{m}$ ) was also presented, which could be useful when longer fibre lengths are required.

\section{References}

[1] J. Noda et al., "Polarization-maintaining fibers and their applications," J. Lightwave Technol., vol.4, pp.10711089 (1986).

[2] M. A. Terrel et al., "Resonant fiber optic gyroscope using an air-core fiber," J. Lightwave Technol., vol. 30, pp.931937 (2012).

[3] J.M. Fini et al., "Polarization maintaining single-mode low-loss hollow-core fibres," Nat Commun., vol. 5, p. 5085. (2014).

[4] F. Poletti, "Nested antiresonant nodeless hollow core fiber," Opt Express, vol. 22, pp.23807-28. (2014).

[5] F.L. Teixeira, et al., "Systematic derivation of anisotropic PML absorbing media in cylindrical and spherical coordinates," IEEE Microw. Guided Wave Lett., vol. 7, pp.371-373. (1997). 